\title{
THE EFFECTS OF SURFACE TREATMENT ON OPTICAL AND VIBRATIONAL PROPERTIES OF STAIN-ETCHED SILICON
}

\author{
Ş. Kalem, D. Göbelek, R. Kurtar, Z. Misırlı \\ TLBITAK, Marmara Research Center, P.K. 21, 41470 Gebze, Kocaeli, Turkey. \\ A. Aydınlı and R. Ellialtıoğlu \\ Bilkent University, Department of Physics, 06533 Ankara, Turkey.
}

\begin{abstract}
The effects of surface treatment on optical and vibrational properties of porous silicon (por-Si) layers grown on p-type Si wafers by electroless etching technique utrt sluditd by FTIR spectroscopy and phololuminescence (PL). The results indicate a correlation between the $P L$ intensity and the strength of the absorption bands induced by multihydride complexes ( $\mathrm{SiH}_{n}, n \geq 2$ ). However, similar correlation was also established for monohydride species as evidenced from the layers containing no multihydrides. Furthermore, a new band is observed at $710 \mathrm{~cm}^{-1}$ and assigned to multihydrides suggesting a $n \in w$ local bonding environment in these layers.
\end{abstract}

Visible light emission from electrochemically modified surfaces of Si wafers has stimulated research since there are ample possibilities of applications (1). It was demonstrated that the stain etching (electroless) in HF solutions also yield the por-Si layers at Si surfaces (2). Recent studies have suggested several reasons such as quantum size effect (1), chemical quantum confinement in siloxene-like structures (3) and hydride excitation (4) as possible explanation for light emission. Despite extensive studies, the precise physical origin of the observed luminescence is not well understood and numerous investigations are still being made in order to bring more insight into the mechanism of the light emission.

Si wafers (p-type) with both (100) (2-20 $\Omega . \mathrm{cm})$ and (111) (10.5-19.5 $\Omega . \mathrm{cm})$ orientations have been subjected to stain etching in $\mathrm{HF}: \mathrm{HNO}_{3}: \mathrm{H}_{2} \mathrm{O}$. Combinations of different type of surface cleaning, etching and post-treatment were used which resulted in layer thicknesses of about $0.3 \mu \mathrm{m}$ as evidenced from cross-sectional SEM micrographs. The saturation in thickness may be attributed to low energy carriers generated during etching in the absence of external fields. Information about hydrogenation and the presence of different species at the surfaces has been obtained from FTIR measurements. PL was excited by an unfocused Ar-ion laser using both $488 \mathrm{~nm}$ and $514 \mathrm{~nm}$ lines at $300 \mathrm{~K}$ using standard detection system which was calibrated to the spectral response of detector.

The results of the PL measurements are summarized in Table 1 for a variety of samples. As it is shown in this table, the emission band is located around $650 \mathrm{~nm}$ with approximately $100 \mathrm{meV}$ bandwidth $(\Delta \mathrm{E})$. This PL cncrgy would corresponds to a cluster size of $20 \AA-30 \AA$ in diameter (mesoporous region), if the size effects are considered in quantum dots. A slight red or blue shift of the peak position occurs depending on the preparation conditions. The blue shift in PL is also confirmed by the shift at the optical absorption edge with respect to crystalline silicon. The PL degradation is observed due 
to strong laser irradation ( $\geq 100 \mathrm{~mW}$ ). But, the PL intensity only decreases without any shift in the spectra after exposure to laser light, suggesting probably that the intrinsic properties are not disturbed due to local heating. Prolonged etching and ultrasonic treatment yielded a slight red shift in PL peak position, a narrowing in PL band and an increase in PL intensity regardless of doping and crystal orientation [except slight blue shift in (111) oriented samples]. In Table 1, the characteristics of PL spectrum of a por-Si taken after 75 minutes in $25 \%$ HF (sample no. \# 12) is shown. This pore widening experiment resulted in a blueshifted weak emission which is in contradiction with the previous report (4). The weak emission in this case can be attributed to excessive removal of oxides which probably leads to an abundant non-radiative recombination centers around nanocrystalline structures.

Typical FTIR vibrational spectra are shown in Figure 1 for layers grown in different electroless etching solutions as function of time and concentration. FTIR bands are located at around $2300-2000 \mathrm{~cm}^{-1}$ (stretching modes), $1000-800 \mathrm{~cm}^{-1}$ (bending modes), $750-600 \mathrm{~cm}^{-1}$ (wagging modes) associated with $\mathrm{Si}_{-} \mathrm{H}_{n}(\mathrm{n} \geq 1)$ bondings. In addition to these vibrations there is a large band at $1070 \mathrm{~cm}^{-1}$ with a shoulder at 1140 $\mathrm{cm}^{-1}$ due to heavy oxidation ( $\mathrm{Si}-\mathrm{O}-\mathrm{Si}$ stretching vibrations). TO modes are located at energies lower than $600 \mathrm{~cm}^{-1}$. In our layers, the band at $2200 \mathrm{~cm}^{-1}$ is the most important and dominant feature among others in this region unlike previous observations made for both anodic and non-anodic por-Si. This vibration is likely associated with multihydride species with one or more oxygen atoms backbonded to $\mathrm{Si}$ from analysis based on earlier studies (5). We find that the band at $870 \mathrm{~cm}^{-1}$ together with the shoulder at $840 \mathrm{~cm}^{-1}$ is the most important band and assigned to multihydrides $\left(\mathrm{Si}-\mathrm{H}_{2}\right)(5)$. We always observe an intense band at $630 \mathrm{~cm}^{-1}$ and a shoulder at $665 \mathrm{~cm}^{-1}$, which are assigned to $\mathrm{Si}-\mathrm{H}$ and $\mathrm{Si}-\mathrm{H}_{2}$ wagging-bending vibrations. In addition, a new band at $710 \mathrm{~cm}^{-1}$ was observed whose strength increases with etching time and dominates this region with increasing $\mathrm{HNO}_{3}$ concentration [Figure 1(b)]. To our knowledge, this is the first observation of the band at $710 \mathrm{~cm}^{-1}$ in por-Si. The correlation in strength between this band and those at 840,870 and $2200 \mathrm{~cm}^{-1}$ suggests that it is associated with multihydrides. The broad absorption band around $460 \mathrm{~cm}^{-1}$ is attributable to TO phonon modes of silicon and

TABLE 1

Summary of the photoluminescence measurements in por-Si layers grown by electroless etching in a variety of solutions. $\lambda_{p}$ : PL peak position, $\Delta \mathrm{E}$ : full width at half maximum.

\begin{tabular}{cccccc}
\hline Sample & $\begin{array}{c}\lambda_{p} \\
(\mathrm{~nm})\end{array}$ & $\begin{array}{c}\Delta \mathrm{E} \\
(\mathrm{meV})\end{array}$ & $\begin{array}{c}\text { Solution } \\
\left(\mathrm{HF}: \mathrm{HNO}_{3}: \mathrm{H}_{2} \mathrm{O}\right)\end{array}$ & $\begin{array}{c}\text { Intensity } \\
(\mathrm{a} . \mathrm{u})\end{array}$ & $\begin{array}{c}\text { Etch Time } \\
(\mathrm{min})\end{array}$ \\
\hline \#2(100) & 647 & 444 & $(4: 1: 5)$ & 990 & 5 \\
$\# 3(100)$ & 657 & 386 & $(4: 1: 5)$ & 9750 & 10 \\
$\# 12(100)$ & 632 & 366 & $(4: 1: 5)$ & 1300 & 5 \\
$\# 15(111)$ & 653 & 419 & $(4: 1: 5)$ & 600 & 2 \\
$\# 18(111)$ & 645 & 400 & $(4: 1: 5)$ & 5400 & 10 \\
$\# 20(111)$ & 662 & 416 & $(4: 2: 5)$ & 725 & 2 \\
$\# 21(111)$ & 659 & 450 & $(4: 2: 5)$ & 5900 & 10 \\
$\# 23(111)$ & 666 & 413 & $(2: 1: 5)$ & 1025 & 2 \\
\hline
\end{tabular}



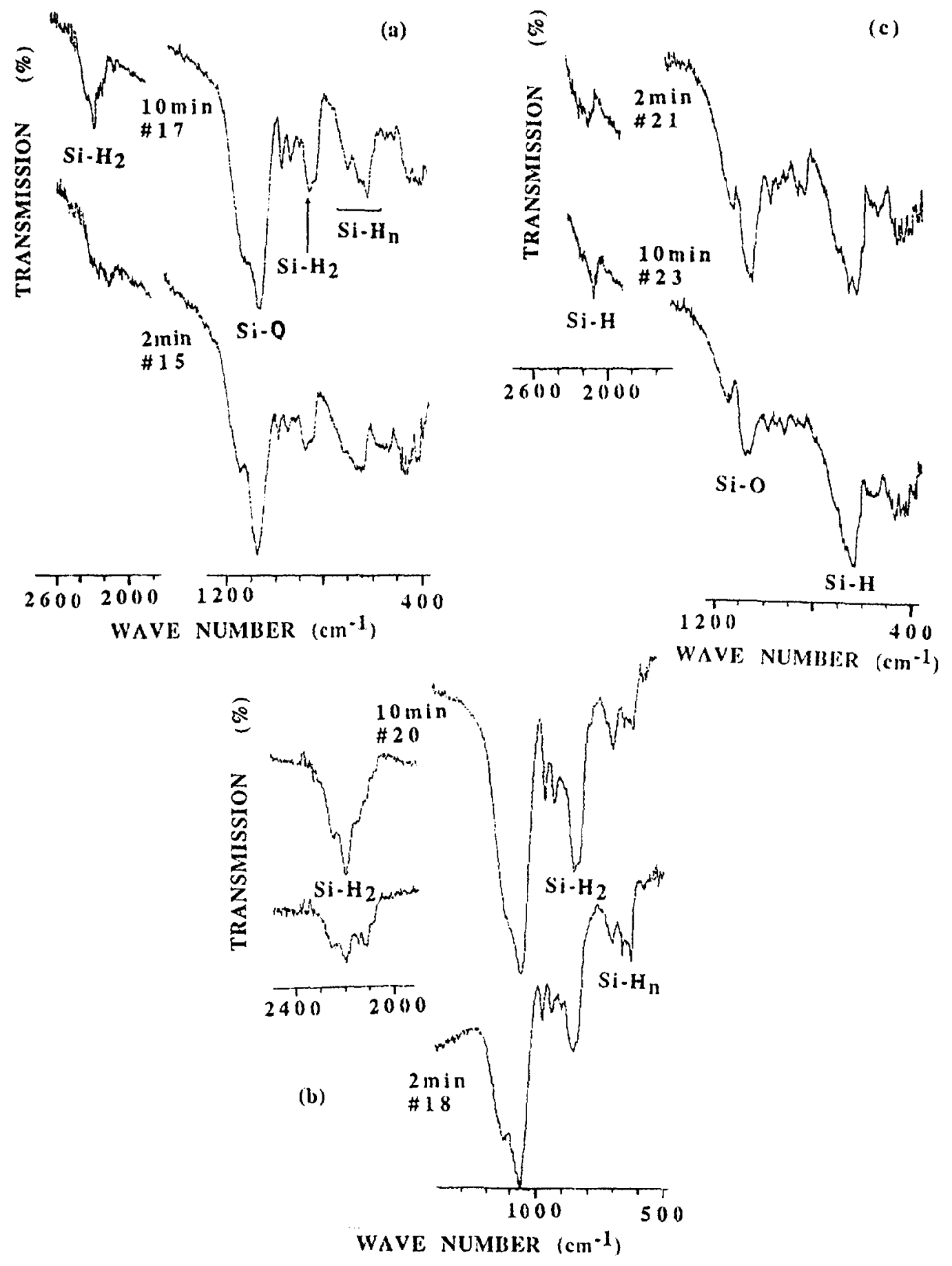

Figure 1. Time evolution of FTIR transmission spectra of por-Si layers grown by stainetching on Si. (a) 4:1:5 (\# 15, \#17), (b) 4:2:5 (\#18, \# 20) and (c) 2:1:5 (\#21, \#23). 
Si-O-Si bending vibrations.

Upon close examination of FTIR spectra [Figure 1(a) and Figure 1(b)] and PL data (Table 1), one finds a good correlation between the PL intensity and the strength of multihydride related bands. The layers grown in both etching solutions (4:1:5 and 4:2:5), the modes (2200, 870 and $710 \mathrm{~cm}^{-1}$ ) associated with the multihydrides dominate the spectral regions of interest. We see also that the predominance of these bands over others increases further with the etching time. This evolution in FTIR bands is followed by an increase in the PL intensity. This situation may be indicative of an increased number of luminescent chemical species or an increased number of Si nano-clusters. The vibrational properties of the layers grown in 2:1:5 solution indicate somewhat different behavior [Figure 1(c)]. For these layers, the monohydride related vibrations dominate spectral regions of interest $\left(2120\right.$ and $\left.630 \mathrm{~cm}^{-1}\right)$. There is a weak multihydride absorption band at $870 \mathrm{~cm}^{-1}$ which disappears almost completely with the etching and the strength of the monohydride related bands increases at 630 and $2120 \mathrm{~cm}^{-1}$. Also, in the spectra of these layers, the oxygen band is very well resolved into two bands $\left(1060\right.$ and $\left.1150 \mathrm{~cm}^{-1}\right)$. The splitting was considered to be the consequence of the ordering of oxygen atoms which is responsible for the luminescence properties in planar siloxene structures (3). However, as it is shown in Table 1, PL intensity is relatively low in these layers. From the analysis of FTIR spectra, we find also that the amount of oxygen in the layers reaches a maximum after a certain time of etching and then decreases for all the samples except those grown in $4: 2: 5$ solution where it increases and then remains constant for the time range explored. If we combine these trends of oxidation with the results of PL summarized in Table 1, we find that the broadening of PL band can be attributed to an increase in oxidation. However, we rule out the possibility of attributing the origin of these changes to the presence of a-Si:O:H like structural phases. Because, in this material, the high energy $\mathrm{PL}$ band is usually accompanied by a defect luminescence at around $1 \mathrm{eV}$, which we do not see in our layers. Previous studies report an effective opening of the band gap with $\mathrm{H}$ content regardless of the type of $\mathrm{Si}-\mathrm{H}$ units (6). In our layers there is no significant change in PL peak position, despite an increase in hydride content. Hence, we suggest that the increase in hydride content might be the result of an increased hydrogenation around $\mathrm{Si}$ clusters or an increase in the number of Si nanocrystallines.

In conclusion, PL intensity in por-Si layers grown by electroless etching is correlated with the increase in intensity of the multihydride related vibrational modes. Nevertheless, PL persists in the layers containing no multihydride complexes. The FTIR spectrum provides evidence for the existence of a new vibrational mode at $710 \mathrm{~cm}^{-1}$ which might bring deeper insight into the understanding of the microstructure of por-Si layers.

The present work was supported by TUBITAK and TWAS (93-116 RG/PHY/AS).

1. V. Lehmann and U. Gosele, Appl. Phys. Lett. $\underline{58}, 856$ (1991).

2. R. Fathauer, T. George, A. Ksendzov, R. Vasquez, Appl. Phys. Lett. 60, 995 (1992).

3. H. Fuchs, M. Stutzmann, M. Brandt, M. Rosenbauer, J. Weber, A. Breitschwerdt, P. Deak, M. Cardona, Phys. Rev. $\underline{B} \underline{48}, 8172$ (1993).

4. S. Prokes, O. Glembocki, V. Bermudez, R. Kaplan, Phys. Rev. $\underline{B} 45,13788$ (1992).

5. G. Lucovsky, J. Yang, S. Chao, J. Tyler, W. Czubatyj Phys. Rev. B 28, 3225 (1983).

6. D. Wolford, B. Scott, J. Reimer, J. Bradley, Physica 117B \& 118B, 920 (1983). 